\title{
Squirmer dynamics near a boundary
}

\author{
Kenta Ishimoto* \\ Research Institute for Mathematical Sciences, Kyoto University, Kyoto 606-8502, Japan \\ Eamonn A. Gaffney \\ Wolfson Centre for Mathematical Biology, Mathematical Institute, University of Oxford, Oxford OX2 6GG, United Kingdom
}

(Received 21 June 2013; published 2 December 2013)

\begin{abstract}
The boundary behavior of axisymmetric microswimming squirmers is theoretically explored within an inertialess Newtonian fluid for a no-slip interface and also a free surface in the small capillary number limit, preventing leading-order surface deformation. Such squirmers are commonly presented as abridged models of ciliates, colonial algae, and Janus particles and we investigate the case of low-mode axisymmetric tangential surface deformations with, in addition, the consideration of a rotlet dipole to represent torque-motor swimmers such as flagellated bacteria. The resulting boundary dynamics reduces to a phase plane in the angle of attack and distance from the boundary, with a simplifying time-reversal duality. Stable swimming adjacent to a no-slip boundary is demonstrated via the presence of stable fixed points and, more generally, all types of fixed points as well as stable and unstable limit cycles occur adjacent to a no-slip boundary with variations in the tangential deformations. Nonetheless, there are constraints on swimmer behavior-for instance, swimmers characterized as pushers are never observed to exhibit stable limit cycles. All such generalities for no-slip boundaries are consistent with observations and more geometrically faithful simulations to date, suggesting the tangential squirmer is a relatively simple framework to enable predications and classifications for the complexities associated with axisymmetric boundary swimming. However, in the presence of a free surface, with asymptotically small capillary number, and thus negligible leading-order surface deformation, no stable surface swimming is predicted across the parameter space considered. While this is in contrast to experimental observations, for example, the free-surface accumulation of sterlet sperm, extensive surfactants are present, most likely invalidating the low capillary number assumption. In turn, this suggests the necessity of surface deformation for stable free-surface three-dimensional finite-size microswimming, as previously highlighted in a two-dimensional mathematical study of singularity swimmers [Crowdy et al., J. Fluid Mech. 681, 24 (2011)].
\end{abstract}

DOI: 10.1103/PhysRevE.88.062702

PACS number(s): 87.17.Jj, 47.63.Gd, 47.63.mf

\section{INTRODUCTION}

Simply examining pond water under a microscope reveals a diversity of swimming microbes, thriving in a low Reynolds number world, where inertia is typically negligible [1-6]. In many experiments, to assure more than a fleeting glimpse of motile microswimmers, the focal plane is typically set adjacent to the coverslip, where microswimmers often accumulate [7-11]. This boundary attraction initiates bacterial biofilms [12], which present major economic challenges and opportunities, such as biofouling, biohydrometallurgy, and bioremediation $[13,14]$. Boundary dynamics also clearly influences sperm motility $[15,16]$, with potential functional consequences, such as reducing the dimensionality of sperm guidance $[17,18]$ and encouraging sperm escape from reservoirs in the isthmus of the estrous mammalian female reproductive tract $[19,20]$.

This prevalence and importance of motile microswimmer boundary induced behaviors has encouraged numerous mechanical studies, from initial explorations of the extent of surface effects $[21,22]$ to the influence of a no-slip boundary on a flagellated bacterium's circling behavior, efficiency, and entrapment $[7,9,23]$. Other examples include the confirmation

\footnotetext{
*ishimoto@ kurims.kyoto-u.ac.jp

†gaffney@maths.ox.ac.uk
}

that detailed flagellar regulation is not required to bring a sperm cell close to a surface [24,25], although a wave form regulation, known as hyperactivation, appears to encourage surface escape [19]. Further studies have also explored the boundary dynamics of idealized swimmers and prospective engineered swimmers [26-28], often via the use of dynamical systems ideas [27], and have for instance illustrated that height oscillations above the surface are possible for inertialess swimmers [27], though this has not been predicted by sperm or bacterial modeling to date.

In addition, a generic and detailed analytical study based on the far-field structure of the flow field has been presented by Spagnolie and Lauga [29]. However, except as part of this generic context, and a brief consideration by Llopis et al. [30], the boundary dynamics of one class of model microswimmer has not yet been considered, namely that of the squirmer [31,32], which induces motility by nonreciprocal surface deformations.

While the swimmer shape and its deformations can be arbitrary, we focus here on axisymmetric bodies with tangential squirming, whereby the swimmer shape remains unchanged but an effective slip velocity is maintained on its surface. Nonetheless, this is a standard abridged model of ciliate motility $[35,36]$, whereby relatively large unicells, with a semiaxis length scale on the order of $50 \mu \mathrm{m}$, are motile due a coating of relatively short actively beating cilia, 
(a)

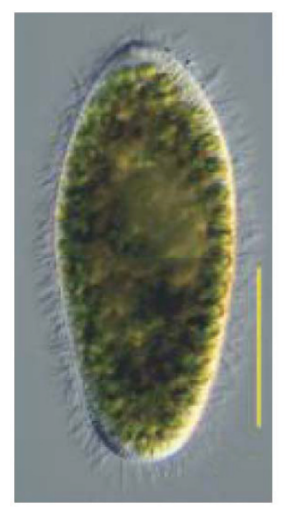

(b)

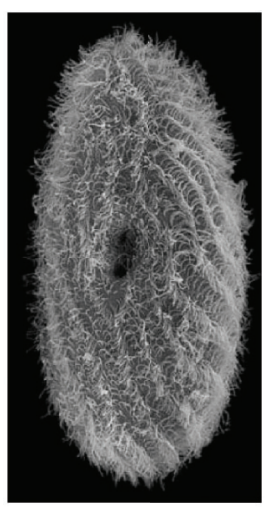

(c)

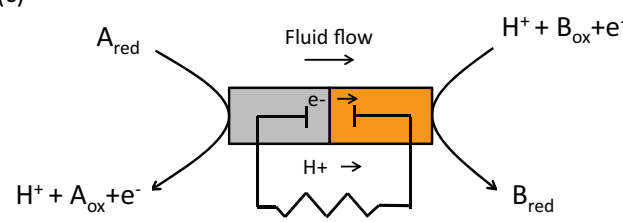

FIG. 1. (Color online) (a),(b) Images of the ciliate, Paramecium (Viridoparamecium) chlorelligerum. (a) An image from flash photomicrography of freely motile specimen after disturbance. Scale bar, $50 \mu \mathrm{m}$. (b) A scanning electron microscope image of the dorsal side, illustrating the density of cilia on the cell surface. Reproduced, with permission, from Kreutz et al. [33]. (c) A schematic of a prospective self-electrophoretic propulsion mechanism for a conducting Janus particle within an acidic environment, whereby a slip velocity is induced by the ion flows generated by a simultaneous catalytic oxidation of a fuel present in the solute, $A$, on one side of the particle and a catalytic reduction of a fuel, $B$, on the other. Reproduced, with permission, from Paxton et al. [34].

which drive a fluid flow around the cell, in turn inducing motility [1]; a classic pond water example is Paramecium, as illustrated in Figs. 1(a) and 1(b). Slip-velocity swimming is also used to model colonial algae such as Volvox carteri, [35,37], whose surface flows are driven by flagella, and Janus particles [29], which have the potential to induce slip velocities by many mechanisms, such as self-electrophoresis via asymmetric surface chemical reactions [34], as detailed in Fig. 1(c).

Thus our first objective in this study will be to classify the behavior of tangential squirmers with axisymmetric bodies and relatively simple slip velocities near no-slip boundaries utilizing dynamical system ideas, in particular a phase plane in the angle of attack and height from the surface. By exploiting boundary element methods for the solution of Stokes' inertialess fluid-dynamical equations, which provide high numerical accuracy for low computational costs $[23,29,37,38]$, we will numerically explore phase space, considering fixed points and their bifurcations as parameters vary. We will also investigate whether more complex dynamics such as limit cycle behaviors and multiple fixed points occur, as well as considering surface scattering, enabling an assessment and characterization of how swimming behaviors near surfaces vary in parameter space for this class of tangential squirmers. We note this generally takes us beyond the scope of Spagnolie and Lauga's [29] recent study of swimmer boundary dynamics using far-field analytical approximation, except for aspects of scattering dynamics where the far-field theory is used to provide an independent check of our simulations.

Of particular further interest is that modeling investigations of boundary swimming to date have almost exclusively focused on no-slip boundaries. However, free surface boundary dynamics merit attention in that an air-water interface is an exploitable microenvironment, facilitating sperm accumulation for laboratory studies [39], as well as providing niche resource opportunities for ciliates [40] and strain dissemination for bacteria [41]. However, current modeling has been restricted to idealized two-dimensional studies including surface deformation [26] and a theoretical prediction that flagellated bacterial circling is reversed near a stress-free surface [9], with a subsequent empirical verification [8,41]. The latter in particular relies on the dynamics of the rotating bacterial flagellum and the counter-rotation induced on the cell body, resulting in a far-field torque dipole, which clearly has an extensive influence on swimmer dynamics near surfaces.

Thus our second objective will be to also characterize how squirmer boundary dynamics may differ in the presence of a stress-free surface in parameter regimes characterized by low capillary numbers, i.e., high surface tension, so that surface deformations are negligible [42], simplifying the analysis. The influence of swimmer strokes generating an additional torque dipole on squirmer boundary dynamics will also be explored, given its importance in bacterial surface behavior.

In summary, microswimmers exhibit a diverse array of surface behaviors and differentiating between hydrodynamical and adaptive biological or chemical effects is fraught with difficulty. Hence we explore how dynamical systems principles provide a means of classifying purely hydrodynamical behaviors, both near no-slip and stress-free surfaces for a simple swimmer, the axisymmetric tangential squirmer which is a common model for ciliates, colonial algae, and Janus particles. The suggested prospect that the far-field dynamics of swimmers may allow approximation of surface behaviors [29] indicates that such characterizations, with the inclusion of rotlet dipoles given the prevalence of rotary flagellated swimmers such as bacteria, may be more widely applicable. Thus in our study of squirmer boundary swimming, our final objective will be to suggest predictions of generic behaviors across parameter space that may be investigated in more specialized, geometrically faithful, studies of specific swimmers.

\section{MODEL SWIMMER AND ITS FLUID DYNAMICS}

\section{A. Squirmer}

We consider an axisymmetric inertialess microswimmer with semiaxes $a, c$, initially above a no-slip wall, which generates propulsion by an axisymmetric tangential surface deformation, represented by a tangential slip velocity. The location of the swimmer is given by the height of its center above the wall, $h$, and the angle between its axis of symmetry and the wall, $\varphi \in(-\pi / 2, \pi / 2)$, as illustrated in Fig. 2, noting that a unique symmetry axis exists even for the spherical swimmer, due to the squirming deformations. The squirmer 


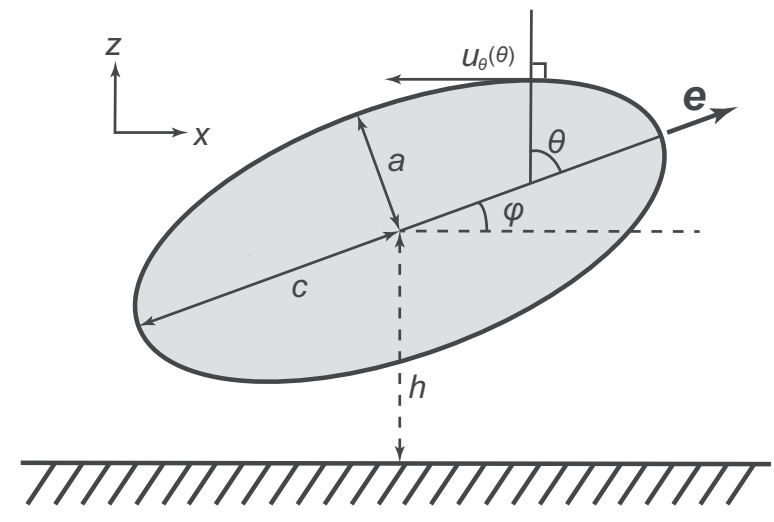

FIG. 2. Schematic picture of an inertialess tangential squirmer, with semiaxes $a, c$, located a height $h$ above a no-slip boundary at an angle $\varphi$, with the axis of symmetry corresponding to the unit vector $\boldsymbol{e}$, directed positively with the swimming direction in the absence of a wall. The polar angle $\theta$ and the axisymmetric tangential slip velocity component, $u_{\theta}(\theta)$, are also depicted. The symmetry of the latter slip-velocity profile ensures the swimmer remains in a plane perpendicular to the wall, which in this paper is taken to be the $x-z$ plane without loss of generality, as depicted.

surface is parametrized by a polar angle, $\theta \in[0, \pi]$, which is the angle between the swimmer surface normal and its axis of symmetry, as shown in Fig. 2, together with an azimuthal angle $\phi \in[0,2 \pi)$, which is the angle around the axis of symmetry, taken clockwise as seen from behind the swimmer.

We first consider a swimmer with a polar slip velocity relative to the body fixed frame. This is represented by a velocity component expressed in terms of an associated Legendre polynomial expansion

$$
u_{\theta}(\theta)=\sum_{n=1}^{\infty} B_{n} V_{n}(\cos \theta),
$$

in the polar tangential direction to the swimmer surface, as depicted in Fig. 2, with

$$
V_{n}(x)=\frac{2 \sqrt{1-x^{2}}}{n(n+1)} \frac{d}{d x} P_{n}(x),
$$

where $P_{n}(x)$ denotes the $n$ th-order Legendre polynomial.

Given the symmetry of this surface velocity, the swimmer moves in a plane, which is perpendicular to the wall and denoted by the $x-z$ plane, as shown in Fig. 2. We nondimensionalize so that the nondimensional viscosity is $\mu=1$, and the volume of the squirmer is given by $(4 \pi / 3) a^{2} c=4 \pi / 3$ and hence the geometry of the swimmer is identified by the aspect ratio $A=c / a$, and all length scales, for instance, the height from the wall, are nondimensionalized by $A^{1 / 3} a$. The swimming velocity of a spherical squirmer $(a=c)$ in free space is simply given by $U=(2 / 3) B_{1}$ on additionally noting an inertialess swimmer is subject to zero net force and torque $[31,32]$.

This highlights how the lower modes of the associated Legendre polynomial expansion strongly dictate the overall behavior of the swimmer. Indeed these modes dominate the far field, which has a particularly simple structure any significant distance from the swimmer, as can be seen in Fig. 3, and thus these modes are likely to dictate initial swimmer-wall

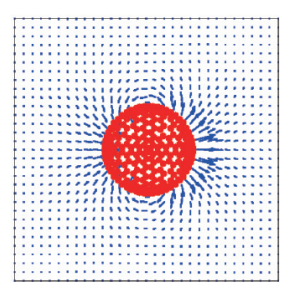

$B_{1}$

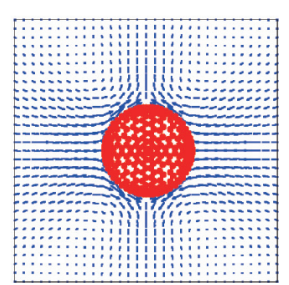

$B_{2}$

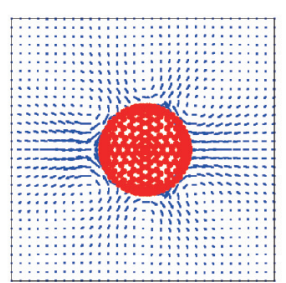

$B_{3}$
FIG. 3. (Color online) Flow field around a spherical swimmer with the direction $\theta=0$ corresponding to the positive horizontal direction for a tangential squirming pattern that possesses only (left) a $B_{1}$ mode, (center) a $B_{2}$ mode, and (right) a $B_{3}$ mode in the associated Legendre polynomial expansion, Eq. (1).

interactions as a squirmer approaches a boundary. Hence, as observed in Spagnolie and Lauga [29], the consideration of the far field often leads to accurate predictions for overall swimmer behavior. Consequently, to limit the dimensionality of parameter space that needs to be considered, we focus on the first three modes of expansion (1) in our explorations. Without loss of generality, we hereafter fix $B_{1}=1.5$ which generates a unit velocity in the case of a free-space spherical squirmer, which is consequently the velocity scale used in the paper. Thus to complete our characterization of tangential squirming deformations, we introduce two further slip-velocity parameters: $\beta_{2}=B_{2} / B_{1}$ and $\beta_{3}=B_{3} / B_{2}$.

\section{B. Far-field expression and singularity solutions}

The flow field obeys Stokes' equation and can be expressed by the superposition of fundamental singular solutions, i.e., the Stokeslet $\mathrm{G}$, the potential source, $\mathrm{H}$, and their multipoles,

$$
\begin{aligned}
u_{j}(\boldsymbol{y})= & \int d S_{\boldsymbol{x}} \sum_{n=0}^{\infty} \frac{\partial^{n}}{\partial \boldsymbol{x}_{i_{1}} \partial \boldsymbol{x}_{i_{2}} \cdots \partial \boldsymbol{x}_{i_{n}}} \\
& \times\left[\alpha_{i, i_{1}, \ldots, i_{n}} \mathrm{G}_{i j}(\boldsymbol{y}, \boldsymbol{x})+\beta_{i_{1}, \ldots, i_{n}} \mathrm{H}_{j}(\boldsymbol{y}, \boldsymbol{x})\right],
\end{aligned}
$$

where the integral is taken over a surface $\boldsymbol{x}$ located in the exterior of the flow [43]. The Stokeslet is also called the Oseen tensor and given by

$$
\mathrm{G}_{i j}(\boldsymbol{x}, \boldsymbol{y})=\frac{\delta_{i j}}{r}+\frac{r_{i} r_{j}}{r^{3}},
$$

where $\boldsymbol{r}=\boldsymbol{y}-\boldsymbol{x}$ and $|\boldsymbol{r}|=r$. Similarly, the potential dipole is given by

$$
\mathrm{H}_{i}(\boldsymbol{x}, \boldsymbol{y})=\frac{r_{i}}{r^{3}},
$$

and it arises in the multipole expansions due to the presence of a boundary.

The flow far from the body is expressed by the first few modes, which are often used in the discussions of the fluid interaction of microswimmers, noting that both strengths of the Oseen tensor and the source singularity must be zero due to the free-force condition and the volume conservation of the swimmer. In particular, for an axisymmetric swimmer the flow 
field is of the form

$$
u_{l}=\alpha e_{i} e_{j} \mathrm{G}_{i j l}^{D}+\beta e_{i} \mathrm{H}_{i l}^{D}+\gamma e_{i} e_{j} e_{k} \mathrm{G}_{i j k l}^{Q}+O\left(|r|^{-4}\right),
$$

where the upper suffices $D$ and $Q$ respectively denote the dipole and quadrupole of each singularity, and the vector $\boldsymbol{e}$ is a unit vector along the swimmer's axis of symmetry, in the same direction as the swimmer motion in the absence of a wall.

Since the swimmer geometry becomes irrelevant for the far-field velocity, the strengths of the singularities in (6) can be evaluated from the far-field expression around the spherical squirmer even though the swimmer is generally spheroidal. Compared with the flow around the spherical squirmer [31], the strengths are respectively found to be

$$
\begin{aligned}
& \alpha=-\frac{3}{4} \beta_{2}, \\
& \beta=\frac{1}{2}-\frac{1}{8} \beta_{3}, \\
& \gamma=-\frac{5}{16} \beta_{3} .
\end{aligned}
$$

The signature of $\alpha$ is used to classify the slip-velocity of the swimmer; in particular, a swimmer with positive $\alpha$ is a pusher, while negative $\alpha$ corresponds to a puller [29]. Microorganisms with tail-like flagella behind the cell, according to the direction set by the overall cell movement, such as bacteria and spermatozoa, can be categorized as pushers. Pullers may be seen in swimmers with their flagella ahead of the cell body, for instance, Chlamydomonas and Leishmania promastigotes. Indeed, $\beta_{2} \sim 1$ is reported for Escherichia coli, whilst $\beta_{2} \sim 0$ for Volvox carteri and artificially created squirmers like a Janus particle, and $\beta_{2} \sim 1$ for the algae genus Chlamydomonas [44]. The potential dipole term, which is a fundamental solution for the Laplace equation of potential flow arises due to the presence of a boundary, while the Stokes quadrupole term typically reflects a fore-aft asymmetry of the swimmer. For instance, the idealized, elongated, bacterial-like swimmers of Spanolie and Lauga [29] have negative $\gamma$, with $|\gamma|<20$, whilst spermatozoa also generate nontrivial values of $\gamma$ due to their fore-aft asymmetry [45].

\section{Dynamical systems and time-reversal symmetry}

Given the surface velocity and the geometric aspect ratio, the force and the torque exerted on the swimmer are completely determined by the configuration of the body, i.e., the distance from the wall $h$ and the direction of the symmetry axis $\varphi$. Therefore, the inertialess Stokes dynamics can be described in terms of the two-dimensional dynamical system,

$$
\begin{aligned}
& \dot{h}=F_{h}(h, \varphi), \\
& \dot{\varphi}=F_{\varphi}(h, \varphi),
\end{aligned}
$$

where the functions $F_{h}$ and $F_{\varphi}$ can be numerically obtained from Stokes' equation. Determining the fixed points of the dynamical system (8) and their linear stability, by considering the associate fixed-point eigenvalues, is fundamental as a stable fixed point of (8) may be regarded as a mathematical expression of stable boundary swimmer behavior.

Furthermore, the dynamical system (8) possesses a timereversal symmetry under the change of variables,

$$
\begin{aligned}
t & \rightarrow-t, \\
\varphi & \rightarrow-\varphi,
\end{aligned}
$$

$$
\begin{aligned}
& \beta_{2} \rightarrow-\beta_{2}, \\
& \beta_{3} \rightarrow \beta_{3},
\end{aligned}
$$

which demonstrates that the dynamical behavior due to a slip velocity with parameters $\left(\beta_{2}, \beta_{3}\right)$ is the same as a time-reversed slip velocity for the dual swimmer with parameters $\left(-\beta_{2}, \beta_{3}\right)$. Hence the behavior of pushers can be understood from that of pullers. For instance, suppose that there exists a stable fixed point $\left(h^{*}, \varphi^{*}\right)$ for a given slip velocity $\left(\beta_{2}, \beta_{3}\right)$. The symmetry (9) then implies that $\left(h^{*},-\varphi^{*}\right)$ is also a fixed point for the dual swimmer with $\left(-\beta_{2}, \beta_{3}\right)$, though stability is lost due to the time reversal in the duality.

\section{Numerical scheme}

The single-layer boundary element method for an incompressible inertialess flow of a Newtonian fluid is used to compute the swimming trajectory. This is founded on an expression for the velocity field as an integral over the surface of the swimmer $S$ [46],

$$
u_{i}(\boldsymbol{x})=-\frac{1}{8 \pi \mu} \int_{S} \mathrm{G}_{i j}\left(\boldsymbol{x}, \boldsymbol{x}^{\prime}\right) q_{j}\left(\boldsymbol{x}^{\prime}\right) d S_{\boldsymbol{x}^{\prime}},
$$

where $\boldsymbol{q}$ is a surface traction given by $\boldsymbol{q}=\boldsymbol{f}-\boldsymbol{f}_{\text {int }}$ with $\boldsymbol{f}, \boldsymbol{f}_{\text {int }}$ respectively denoting the surface tractions due to the external and internal Newtonian flows associated with the swimmer surface velocities. This formalism entails that $\boldsymbol{q}$ possesses a gauge degree of freedom, namely an additive constant of the surface normal-this is removed by setting $\int_{S} \boldsymbol{q} \cdot \boldsymbol{n} d S_{\boldsymbol{x}^{\prime}}$ to zero. The Green function $\mathrm{G}$ is the Stokeslet in the presence of an infinite no-slip rigid wall [47], known as the Blakelet, or in the presence of an infinite free-slip wall, according to the problem under consideration. For the integral kernel in the case of the free-slip wall, only the image singularity is required (see, for example, Appendix B of [29]), and the Green function is given by

$$
\mathrm{G}_{i j}^{\text {slip }}\left(\boldsymbol{x}^{\prime}, \boldsymbol{x}\right)=\mathrm{G}_{i j}\left(\boldsymbol{x}^{\prime}, \boldsymbol{x}\right) \pm \mathrm{G}_{i j}\left(\boldsymbol{x}^{\prime}, \boldsymbol{x}^{*}\right)
$$

where $\boldsymbol{x}^{*}$ is the mirror image of $\boldsymbol{x}$ with respect to the infinite wall, $\boldsymbol{x}^{*}=\boldsymbol{x}-2 h \hat{\boldsymbol{z}}$, and $\boldsymbol{z}$ is an unit vector along the $z$ axis. The sign in (10) is taken to be positive when $i=x, y$ and negative otherwise.

The boundary condition on the surface of the swimmer, $S$, is a continuity of velocity, so that the fluid velocity matches the local surface velocity of the swimmer. The position of the swimmer surface $\xi$ in the laboratory frame can be written as $\boldsymbol{\xi}=\boldsymbol{X}+\mathrm{B} \cdot \boldsymbol{\xi}^{\prime}$ (Fig. 4), where $\boldsymbol{X}$ is the origin of the body frame in the laboratory frame, corresponding to the center of the swimmer, $\boldsymbol{\xi}^{\prime}=\boldsymbol{x}^{\prime}-\boldsymbol{X}$ is the surface position in the body frame, and, following [25], B is a set of column basis vectors of the body frame. Let $\boldsymbol{U}$ and $\boldsymbol{\Omega}$ be the translational and rotational velocity of the origin of the laboratory frame $\boldsymbol{X}$. Then the surface velocity of the swimmer in the laboratory frame is

$$
\boldsymbol{v}(\boldsymbol{\xi})=\boldsymbol{U}+\boldsymbol{\Omega} \times \boldsymbol{\xi}^{\prime}+\mathrm{B} \cdot \dot{\boldsymbol{\xi}}^{\prime} .
$$

The boundary condition that the velocity vector field does not slip relative to the swimmer surface deformation, 


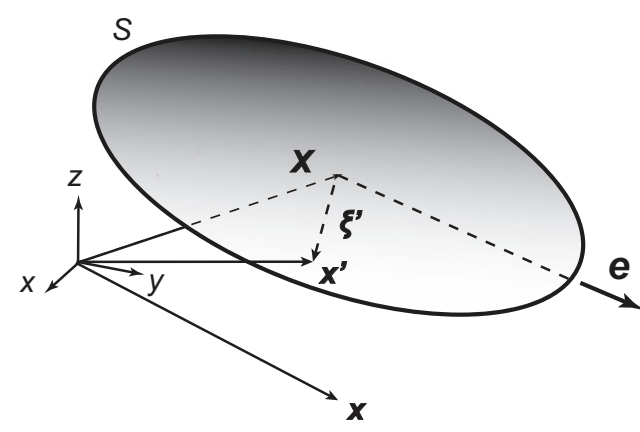

FIG. 4. Schematic picture of the swimmer configuration-its surface and axis of symmetry are respectively given by $S$ and $\boldsymbol{e}$, with the latter's direction dictated by the swimming direction in the absence of a wall. A laboratory reference frame has Cartesian coordinates $x, y, z$ and the position vector of the swimmer's center, a point on its surface and an external field point, relative to this frame, are respectively denoted by $\boldsymbol{X}, \boldsymbol{x}^{\prime}$, and $\boldsymbol{x}$. Analogously, a point on the swimmer's surface relative to a body fixed frame with origin at the swimmer center, is denoted $\xi^{\prime}$.

$\boldsymbol{v}\left(\boldsymbol{x}^{\prime}\right)=\boldsymbol{u}\left(\boldsymbol{x}^{\prime}\right)$, thus becomes

$$
\begin{aligned}
u_{j} & =U_{i}+\epsilon_{i j k} \Omega_{j} \xi_{k}^{\prime}+\mathrm{B}_{i j} \dot{\xi}_{j}^{\prime} \\
& =-\frac{1}{8 \pi \mu} \int_{S} \mathrm{G}_{i j}\left(\boldsymbol{x}^{\prime}, \boldsymbol{x}^{\prime \prime}\right) q_{j}\left(\boldsymbol{x}^{\prime \prime}\right) d S_{\boldsymbol{x}^{\prime \prime}}
\end{aligned}
$$

Noting that the force and torque generated on the swimmer by the internal force, $\mathbf{f}_{\text {int }}$, are both zero [46], the total force and torque balance equations can be written in the form

$$
\int_{S} \boldsymbol{q}\left(\boldsymbol{x}^{\prime}\right) d \boldsymbol{S}_{x^{\prime}}=\int_{S}\left(\boldsymbol{x}^{\prime}-\boldsymbol{X}\right) \times \boldsymbol{q}\left(\boldsymbol{x}^{\prime}\right) d \boldsymbol{S}_{\boldsymbol{x}^{\prime}}=\mathbf{0} .
$$

The unknown variables $\boldsymbol{q}\left(\boldsymbol{x}^{\prime}\right), \boldsymbol{U}$, and $\boldsymbol{\Omega}$ are then obtained by solving the linear problem (12), subject to the balance equations (13).

For mesh generation, we have employed the BEMLIB library accompanying [43]. The number of mesh elements used here is $N=512$ or $N=2048$, depending on the accuracy that is required. At each time point, the problem then reduces to the solution of a dense matrix equation in $3 N+6$ unknowns, analogously to [37]. This yields the body velocity and angular velocity at each time point in terms of the surface deformations, which is sufficient to find phase-space fixed points. When swimmer trajectories are required, there is a subsequent time marching which proceeds via a Heun scheme, as presented by Smith et al. [25], and elements of these simulations have been performed using the cluster computing system within Research Institute for Mathematical Sciences (RIMS), Kyoto University.

Once the distance between the swimmer surface and the substrate is of the order of $0.1 \mu \mathrm{m}$ or less, additional interaction forces manifest between a bacterial cell and the boundary, which are highly dependent on the details of the substrate, the swimmer surface, and the solution media [48]. We do not consider such molecular-level complexities here and our computation stops if the swimmer approaches this close to the wall and we simply conclude that under such circumstances hydrodynamics brings the swimmer up to the wall-drawing more detailed conclusions would require caution.

\section{DYNAMICS IN PHASE SPACE}

\section{A. Swimming near a no-slip boundary}

We proceed to numerically examine the fixed points for height, $h$, and angle, $\varphi$, using the standard Newton-Raphson method, which calls the boundary element solver to determine values of the functions $F_{h}$ and $F_{\varphi}$ in Eq. (8), in turn allowing the roots of $F_{h}=F_{\varphi}=0$ to be found. The results are illustrated in Fig. 5-the reflection symmetry and antisymmetry with respect to $\beta_{2}$ is readily apparent in the phase diagram and is a consequence of the time-reversal symmetry of this system.

Note that by the consideration of the free space solutions, we anticipate straight-line swimming trajectories as $h \rightarrow \infty$ due to negligible fluid interactions with the wall; such regions of phase space are neglected below since the dynamics is trivial. In particular, since the time variation of $h$ and $\varphi$ becomes quite small when the swimmer is far from the wall $(h \gg 1)$, so that $F_{h}$ and $F_{\varphi}$ vary only weakly, the accuracy requirements in finding any prospective large- $h$ fixed point become extremely demanding. Furthermore, such a fixed point can be regarded as physically meaningless in terms of swimmer-boundary attraction. Thus we introduce a cutoff
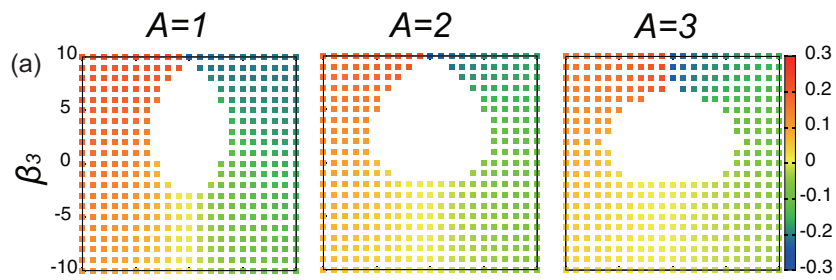

(b)
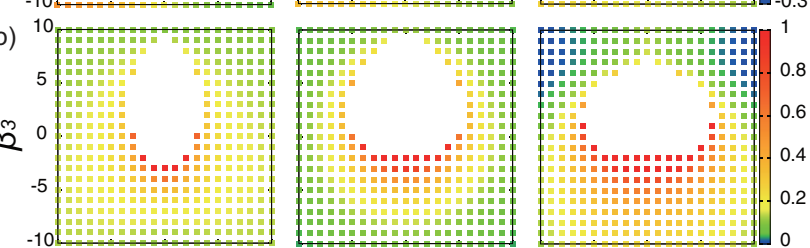

(c)
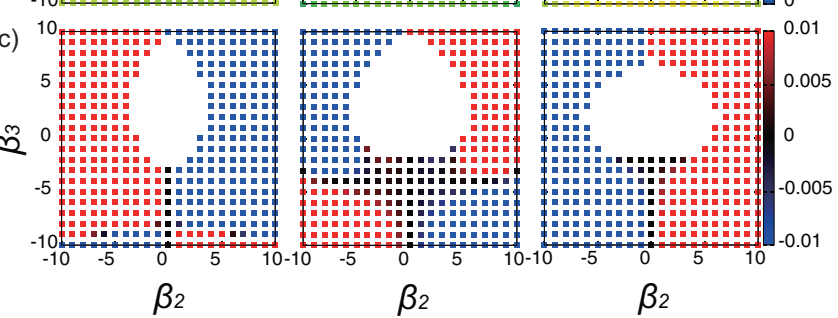

FIG. 5. (Color online) Plots detailing the location and stability properties of phase-space fixed points for a given slip-velocity parametrization $\left(\beta_{2}, \beta_{3}\right)$, with different swimmer aspect ratios $A=$ $1,2,3$ and the presence of a no-slip boundary. If no color is plotted there is no fixed point for the given parameter values. The upper row (a) presents the steady-state angle between the axis of symmetry and the wall, $\varphi^{*}$, associated with each fixed point in units of radians. When $\beta_{2} \sim 0$ and $\beta_{3}>0$, there are multiple fixed points, though we only plot the one with the most negative $\varphi$ when $\beta_{2} \geqslant 0$, and the most positive otherwise; these fixed points are nodes with nearby adjacent saddles, as illustrated in Fig. 6 and discussed further in the text. The central row (b) depicts the shortest distance between the swimmer and the wall for the plotted fixed point. The bottom row (c) gives the largest real part of the linear stability eigenvalues associated with the plotted fixed point. 
for the height of the fixed point at $h_{\text {far }}=15$, i.e., a scale of 15 swimmer lengths from the wall, where the squirmer-wall interactions are assured to be very small.

Achieving accuracy for any prospective fixed point very close to the wall is also computationally extremely demanding, due to difficulties in sufficiently resolving the singularities and their images when the swimmer surface is extremely close to the wall [23]. In particular, the boundary element scheme and discretization used here lose accuracy when the distance $d$ between the wall and the nearest point of the swimmer, which is given by $d=h-A^{-1 / 3} \cos (\varphi) \sqrt{1+A^{2} \tan ^{2}(\varphi)}$, decreases below 0.01 nondimensional units. This is on the scale of 0.01 of the squirmer semiaxis: even for a swimmer as large as the ciliate depicted in Fig. 1 this is about $0.2 \mu \mathrm{m}$, which is approaching a scale necessitating the inclusion of nonhydrodynamic, molecular-level, details in the squirmersurface wall interactions. Thus, once more, drawing any quantitative conclusions relying on the modeling predictions in this dynamical regime requires caution.

In Fig. 5, there is a region at the center of the phase diagram where no fixed points exist. As the slip-velocity parameters $\left(\beta_{2}, \beta_{3}\right)$ approach this region the steady-state height, $h^{*}$, grows rapidly as can be seen in the middle row of Fig. 5. Thus, for example, the fully activated Janus swimmer, which is classified by $\beta_{2}=\beta_{3}=0$ (Fig. 5 [29]) does not have a fixed point near a wall.

Now consider a swimmer with $\beta_{2}=0, \beta_{3}<0$ - there is a fixed point with zero steady-state angle, $\varphi^{*}=0$, and pure imaginary eigenvalues. More generally, if there is a fixed point with $\varphi^{*}=0$, the real part of the eigenvalues must be zero due to the time-reversal symmetry discussed above. Such a fixed point is a center, with the linear theory prediction that phase-space trajectories form closed loops sufficiently close to the fixed point. While in general this need not reflect the behavior of the full nonlinear system, due to the breakdown of Hartman's theorem, the time-reversal symmetry ensures that such trajectories are indeed closed loops.

Except for $\beta_{2} \sim 0$ with $\beta_{3}$ relatively large, which we discuss later below, there is a single fixed point; in this case, the eigenvalues have nonzero real parts when $\beta_{2} \neq 0$ and thus the fixed point is a phase-plane focus. Consequently, when the real part of the eigenvalue is negative, the swimmer progresses in one direction essentially parallel to the wall, with a damped height oscillation that relaxes to the fixed point on approaching stable swimming and presents a growing unstable oscillation otherwise.

The presence or absence of this stability is highly sensitive to the swimmer aspect ratio $A$, as can be observed from the lowest row of Fig. 5. First of all, let us consider the case of the spherical squirmer with $A=1$. For a swimmer with positive $\beta_{2}$ (a puller), there are two regions with distinct stability behaviors in the figure: an unstable region with smaller $\beta_{3}$ and a stable region with larger $\beta_{3}$. Our computational results show that as the geometric aspect ratio, $A$, is increased from 1 to 2 , some fixed points are lost to infinity and the stable region with $\beta_{2}>0$ is compacted into a region with smaller $\beta_{3}$. There is also a new unstable region for larger $\beta_{3}$ and analogous dual changes occur for $\beta_{2}<0$. With a further increase in the aspect ratio to $A=3$, boundary swimming with $\beta_{2}>0$ is now unstable, in distinct contrast to most of the parameter space for $A=1$. Hence both pushers and pullers can stably swim near a boundary with an appropriate slip-velocity pattern, which is generally contingent on the squirmer geometry. Furthermore, one can also observe that whenever a puller $\left(\beta_{2}>0\right)$ has a stable fixed point, its angle relative to the wall is negative, in contrast to a pusher, reflecting the duality imposed by time-reversal symmetry.

Remarkably, we can find regions containing multiple fixed points when $\beta_{2} \sim 0$ and $\beta_{3}$ is relatively large. These do not contradict time-reversal symmetry as the two steady-state angles and the associated linear stability eigenvalues differ by a minus sign, while the steady-state height is the same. In this parameter regime, with $\beta_{2} \geqslant 0$, only the fixed point associated with the most negative angle is plotted in Fig. 5, whilst the fixed point associated with the most positive angle is plotted for $\beta_{2}<0$. In addition, we observe an adjacent saddle point. The dynamics in this regime is therefore more complicated than a phase-plane focus that we observed above. Thus, to understand swimming behaviors in the presence of these multiple fixed points, and global behavior more generally, the swimmer's overall behavior in phase space also needs to be explored. We therefore numerically examine swimmer trajectories in phase space and, more generally, the $(h, \varphi)$ phase-plane portrait.

First, we consider a parameter regime with multiple fixed points. With $A=1, \beta_{2}=0, \beta_{3}=10$, the phase portrait is plotted in Fig. 6. Nodes are depicted by circles, and the node associated with a negative steady-state angle, $\varphi^{*}$, is unstable in contrast to the node associated with positive $\varphi^{*}$. Two saddle points are also present and close to the nodes so that local to each saddle-node pair the phase portrait is analogous to that of a saddle-node bifurcation. In particular, such fixed-point pairs are not globally stable, as the saddle's unstable manifold drives integral paths away from the fixed points, and thus the associated dynamics can be highly contingent on the approach to the fixed points, even when one of them is locally stable. In particular, stable boundary swimming need not occur, and the

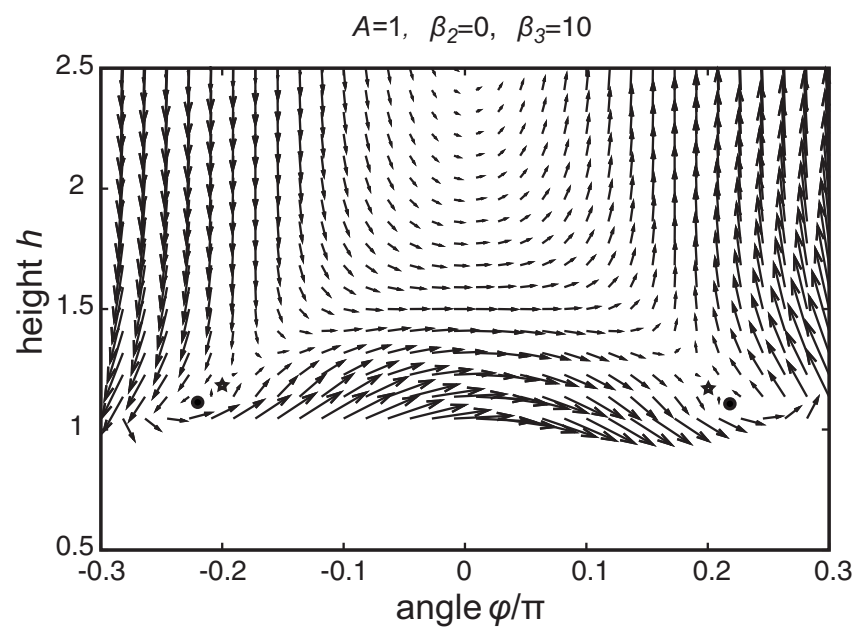

FIG. 6. Phase plane portrait for $A=1, \beta_{2}=0, \beta_{3}=10$ with arrows indicating flow in the dynamical system. Four fixed points can be observed with a duality in the sign of the steady-state angle. The circles indicate two fixed points, one of which at $\left(h^{*}, \varphi^{*}\right)=(1.093,0.231 \pi)$ is a stable node, whereas the other at $\left(h^{*}, \varphi^{*}\right)=(1.093,-0.231 \pi)$ is an unstable node. Also, two saddle points are depicted by stars, at $\left(h^{*}, \varphi^{*}\right)=(1.222 \pm 0.194 \pi)$. 
swimmer can either approach the surface or be scattered from it, depending on initial conditions. However, this dynamical system phase-plane portrait relies on relative fine-tuning as the saddle and node annihilate in a bifurcation as $\beta_{2}$ moves away from zero. Nonetheless, certain aspects of the phase plane are also present even with a single stable fixed point, such as the observation that the swimmer can approach the boundary or escape at sufficiently extreme angles, for example.

We proceed to consider a swimmer's trajectory for a slip-velocity associated with an unstable fixed point. As the slip-velocity parameters $\left(\beta_{2}, \beta_{3}\right)$ are changed along a curve in $\left(\beta_{2}, \beta_{3}\right)$ parameter space where the real part of the eigenvalues transition through zero without a change in the sign of $\beta_{2}$, the fixed point undergoes a Hopf bifurcation. In the case of $A=2$, for instance, when the parameters change from $\left(\beta_{2}, \beta_{3}\right)=$ $(6,-10)$ to $(6,5)$, the stable fixed point becomes unstable at $\beta_{3} \sim-4$. We therefore explore the swimmer dynamics in phase space after the Hopf bifurcation has occurred by employing initial conditions which constitute a perturbation away from the unstable fixed point, and then computing the swimmer trajectory until it approaches the wall or moves away $\left(h \geqslant h_{\text {far }}\right.$ ), or the large time dynamics is revealed. In particular, the Hopf bifurcation is supercritical and a stable limit cycle is observed as highlighted in Fig. 7 for parameters $A=2, \beta_{2}=6, \beta_{3}=-2$. Furthermore, as $\beta_{3}$ further increases, the limit cycle becomes larger and approaches the wall, eventually intersecting it. Thus this trajectory finally becomes globally unstable in the sense that the swimmer is brought sufficiently close to the wall by hydrodynamic interactions that surface-swimmer molecular interaction forces manifest, at which point the model presented here breaks down, as schematically illustrated in Fig. 8. In contrast, when $\beta_{2}$ is negated for this set of parameters, the stability is lost due to the time-reversal symmetry, which entails the appearance of an unstable limit cycle together with a stable fixed point at a subcritical Hopf bifurcation, as reported in [27] for a simple three-sphere swimmer.

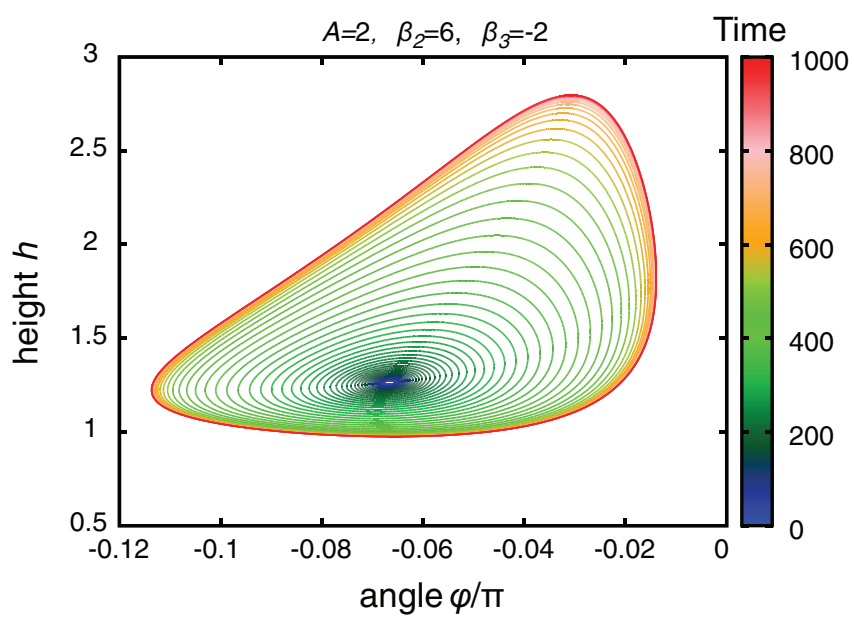

FIG. 7. (Color online) Phase-space trajectory for $A=2, \beta_{2}=$ $6, \beta_{3}=-2$. The color contour shows the time after departure from an initial point adjacent to the unstable fixed point. Clearly, the trajectory ultimately converges to a limit cycle.

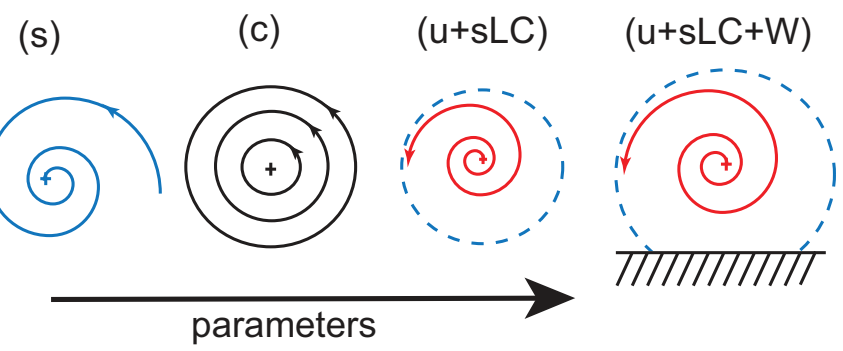

FIG. 8. (Color online) Schematic picture of the Hopf bifurcation and its influence on global behavior in the phase space. A stable fixed point becomes unstable as a parameter varies, together with the appearance of a limit cycle which finally approaches and intersects the boundary. The stability of the fixed point is characterized via (s) stable, (c) nonlinear center, $(\mathrm{u})$ unstable, $(\mathrm{u}+\mathrm{sLC})$ unstable fixed point with a surrounding stable limit cycle, and $(\mathrm{u}+\mathrm{sLC}+\mathrm{W})$ unstable fixed point with a surrounding stable limit cycle that intersects the wall.

\section{B. Swimming near a free surface}

The swimmer near a free surface is investigated analogously, under the assumption that the capillary number is asymptotically small, so that surface deformation is negligible [42]. The angle, $\varphi^{*}$, and distance of closest approach are depicted for fixed points in Fig. 9 as a function of the geometric aspect ratio, $A$, and the slip-velocity parameters $\beta_{2}, \beta_{3}$. Once more the signature of the angle between the axis of symmetry and the wall at the fixed point, $\varphi^{*}$, is determined by the sign of the slip-velocity parameter $\beta_{2}$, though fixed points are now lost into the wall when $\beta_{2}=0$ as the aspect ratio increases, for example, in contrast to observations for a no-slip boundary.
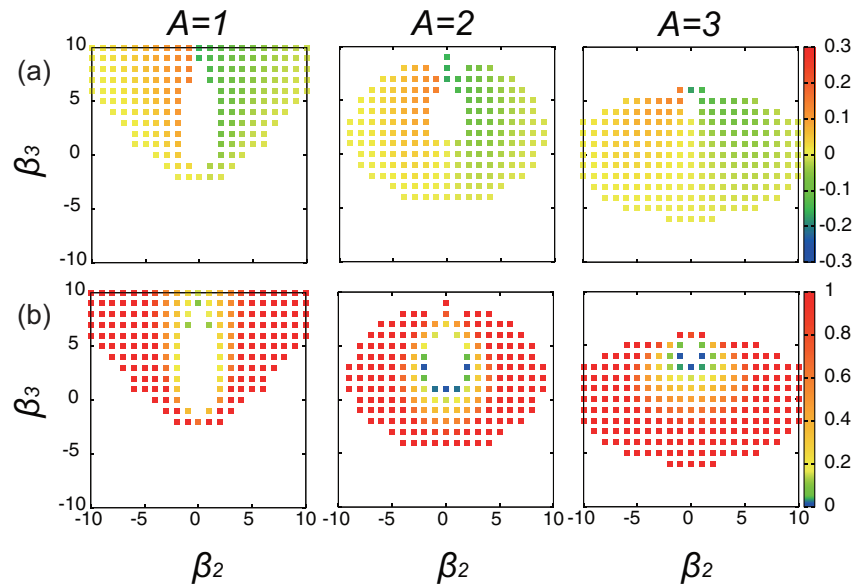

FIG. 9. (Color online) Plots detailing the properties of phasespace fixed points for a given slip-velocity parametrization $\left(\beta_{2}, \beta_{3}\right)$, with different geometric aspect ratios $A=1,2,3$ in the presence of a free-surface boundary at asymptotically small capillary numbers, so that surface deformation is negligible. If no color is plotted there is no fixed point for the given parameter values. The upper row (a) plots the angle, $\varphi^{*}$, of each fixed point and the lower row (b) denotes the shortest distance between the swimmer and the wall at the fixed point. When there are multiple fixed points, all are still saddles though only the one with the most negative $\varphi$ is plotted above when $\beta_{2} \geqslant 0$; otherwise, the fixed point associated with the most positive $\varphi$ is plotted; see text for further details. 


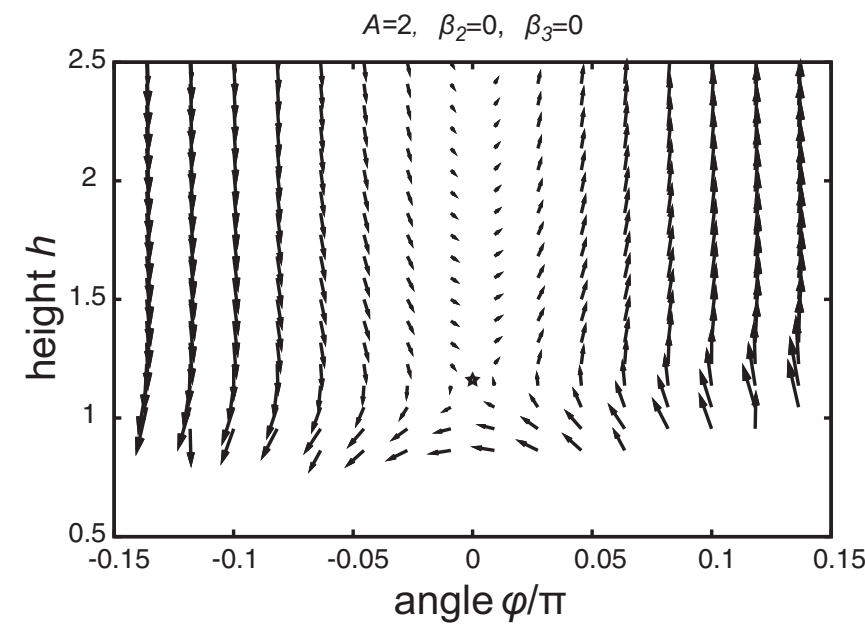

FIG. 10. Phase-plane portrait for an aspect ratio $A=2$, and slipvelocity parameters $\beta_{2}=\beta_{3}=0$ in the presence of a free surface. The star highlights the location of a saddle point at $\left(h^{*}, \varphi^{*}\right)=(1.147,0)$; note that no stable accumulation dynamics is indicated by the phaseplane trajectories.

The most surprising and interesting difference though is the absence of stable fixed points in the same parameter space surveyed for the no-slip boundary case; instead we only find saddle points, demonstrating that the swimmer cannot be stably trapped near the boundary in this substantial region of parameter space.

An illustration of the global behavior in the presence of a free surface boundary is given by the dynamical system flow presented in Fig. 10 for a geometric aspect ratio of $A=2$ and slip-velocity parameters $\left(\beta_{2}, \beta_{3}\right)=(0,0)$ corresponding to a fully activated Janus particle [29]. The only fixed point is highlighted by a star and is a saddle, with no stable boundary swimming. Instead (except on the stable manifolds of the saddle), the swimmer either escapes to infinity or approaches the wall, respectively with a positive and a negative angle $\varphi$. We finally note that multiple saddle points can co-exist with opposite fixed-point angle, $\varphi^{*}$, for a large value of $\beta_{3}$; in such

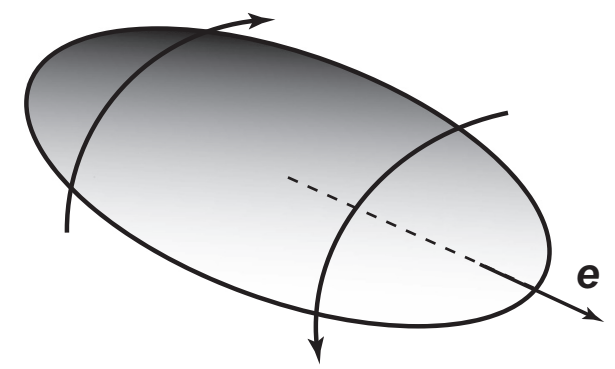

FIG. 12. Schematic picture of a squirmer with an additional rotlet dipole. The illustrated scenario case has a positive orientation and thus possesses positive $\gamma_{2}$.

cases, the squirmer can approach the wall with a positive angle though once more stable boundary swimming is not possible.

\section{SCATTERING BEHAVIOR}

To examine the difference in swimmer behaviors in the absence of fixed points and either a no-slip or a freesurface boundary, we consider scattering trajectories for a spherical swimmer $(A=1)$, initially located at $(x, z)=(0,2)$ and with tangential deformation parameters $\left(\beta_{2}, \beta_{3}\right)=(0,0)$. In Fig. 11(a) the relation between the initial angle $\varphi_{\text {init }}$ and the final angle $\varphi_{\text {fin }}$ for both no-slip and free-surface boundaries are plotted, using squares and triangles. Note that below an initial angle of $\varphi \sim-0.09 \pi$, the swimmer is not scattered by a free surface, whilst scattering occurs for a no-slip boundary until the initial angle is as negative as $\varphi \sim-0.19 \pi$. Furthermore, in Fig. 11(b), where sample trajectories in physical space are plotted for an initial angle of $-0.05 \pi$, there is clearly an extended residence of the swimmer in the vicinity of the free surface during scattering, compared to a no-slip boundary. Finally, in Fig. 11(a), the solid curve represents the far-field approximation expression for scattering from the no-slip boundary, $\varphi_{\text {fin }}=\sqrt{\varphi_{\text {init }}^{2}+1 / 64}$ [29], and clearly agrees with the no-slip boundary computation, providing an independent validation of the numerical simulations.
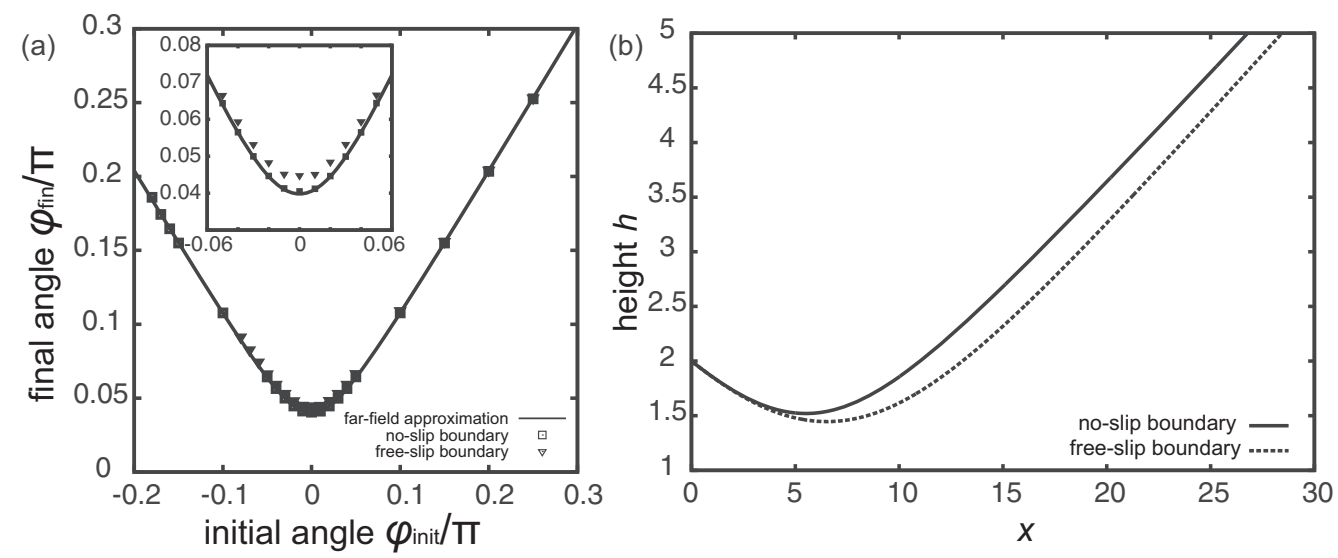

FIG. 11. (a) Plot of the final angle, $\varphi_{\text {fin }}$, in terms of the initial angle, $\varphi_{\text {init }}$, for the scattering trajectory followed by a swimming spherical squirmer with aspect ratio $A=1$ and slip-velocity parameters $\left(\beta_{2}, \beta_{3}\right)=(0,0)$ in the presence of both a no-slip and a free-surface boundary; the far-field theory predictions are also plotted (solid). Inset: a magnified view when the initial angle is near zero. (b) The trajectory when this swimmer is initially located at $(x, z)=(0,2)$ with initial angle $\varphi=-0.05 \pi$ for both a no-slip and a free-surface boundary. 


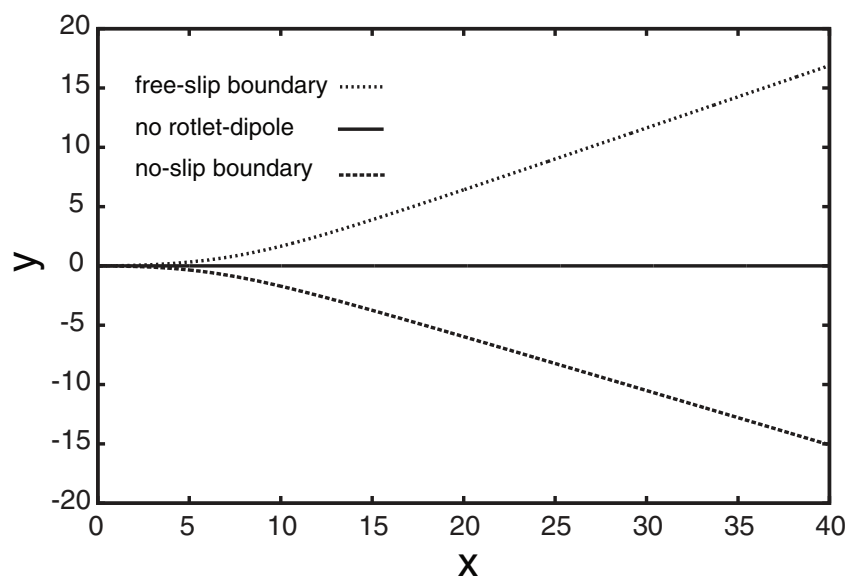

FIG. 13. Swimming trajectories projected into the $x-y$ plane for the spherical squirmer $\left(A, \beta_{2}, \beta_{3}\right)=(1,0,0)$, with and without a positively oriented rotlet dipole, adjacent to a no-slip or a free surface with initial location $(x, y, z)=(0,0,2)$ and direction $\varphi=-0.05 \pi$. The swimmer with $\gamma_{2}=5$ rotates clockwise close to a no-slip boundary and counterclockwise near a free surface, whilst swimming in the absence of a rotlet dipole induces motion only in the $x$-z plane.

\section{SWIMMER WITH A ROTLET DIPOLE}

We proceed to briefly consider a rotary swimmer, such as a bacterium, which is driven by a torque motor rapidly rotating a flagellum or multiple flagella in one direction, and thus inducing a cell body rotation in the opposite direction, as required for conservation of angular momentum. To consider such influences, we allow the squirmer to possess an axisymmetric tangential deformation in the azimuthal direction, with this component of the surface velocity expressed in terms of a stream function $\Psi$, via

$$
u_{\phi}=\frac{\Psi}{r \sin \theta} .
$$

Here the stream function $\Psi$ satisfies

$$
\left[\frac{\partial^{2}}{\partial r^{2}}+\frac{\sin \theta}{r^{2}} \frac{\partial}{\partial \theta}\left(\frac{1}{\sin \theta} \frac{\partial}{\partial \theta}\right)\right]^{2} \Psi=0
$$

and, similar to the polar angle component (1), we introduce a series expression of the surface velocity,

$$
u_{\phi}(\theta)=\sum_{n=1}^{\infty} C_{n} V_{n}(\cos \theta)
$$

Separation of variables gives the azimuthal velocity, via the solution of (15), as

$$
u_{\phi}(r, \theta)=\sum_{n=1}^{\infty} \frac{C_{n}}{r^{n+1}} V_{n}(\cos \theta),
$$

which decays on the scale $O\left(r^{-1}\right)$ in the far field, where the resulting flow can be also expanded in terms of the fundamental singular solution of a point torque, or rotlet, and its multipoles [46]. The rotlet is given by

$$
\mathrm{R}_{i j}=-\frac{\epsilon_{i j k} r_{k}}{r^{3}}
$$

though the leading term in the rotlet multipole expansion must be trivial due to the torque-free condition. Thus the leading term of the far-field velocity due to the azimuthal tangential deformations is given the rotlet dipole, $R_{i j k}^{D}$, and takes the form

$$
u_{k} \sim \tau e_{i} e_{j} \mathrm{R}_{i j k}^{D},
$$

where $\mathbf{e}$ is the axis of symmetry in Fig. 2 and $\tau$ is a constant, measuring the magnitude of the rotary flow and signed via its orientation. It may be determined by comparison with Eq. (17) and is given by $(1 / 3) C_{2}$, with positive $\tau$ corresponding to the front of the cell, towards $\theta=0$, rotating clockwise and the aft rotating anticlockwise when viewed from behind, as depicted in Fig. 12, which corresponds to the chirality of E. Coli, whilst the opposite chirality is exhibited by $R$. Sphaeroides $[49,50]$.

We therefore introduce another slip-velocity parameter, $\gamma_{2}$, defined as $\gamma_{2}=C_{2} / B_{1}$, to incorporate the rotary contribution of the tangential deformation. Its effect only influences the squirmer trajectory near a boundary. In particular, Fig. 13 illustrates the trajectories of the spherical swimmer $\left(A, \beta_{2}, \beta_{3}, \gamma_{2}\right)=$ $(1,0,0,5)$ viewed from above, with initial position $(x, y, z)=$ $(0,0,2)$ and direction $\varphi=-0.05 \pi$. Note that the no-slip boundary case induces anticlockwise circling with a positively oriented rotlet dipole, as also observed in Fig. 14(a), and in the
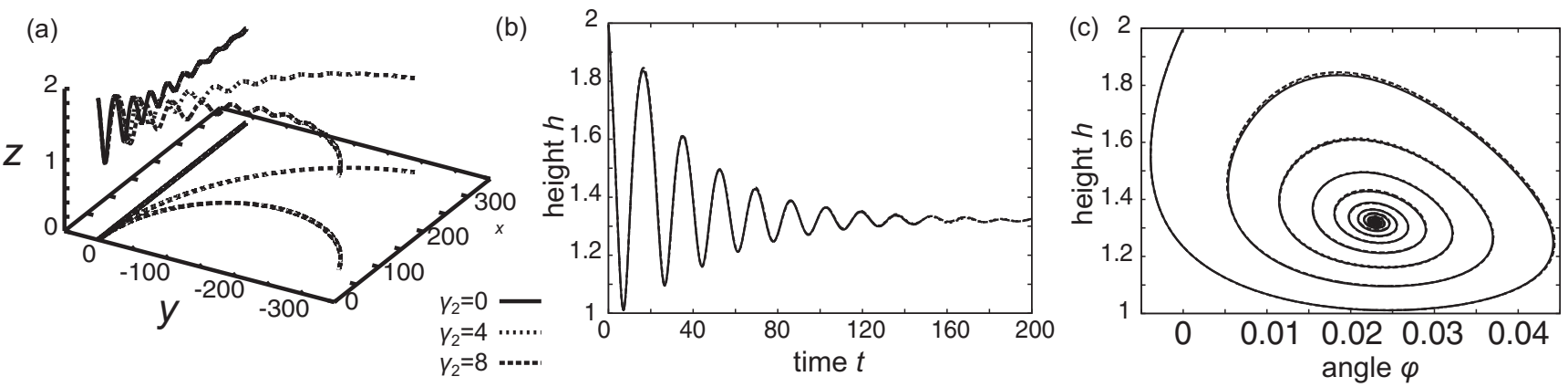

FIG. 14. Trajectory of a squirmer adjacent to a no-slip boundary, with aspect ratio $A=3$, and polar slip-velocity parameters $\left(\beta_{2}, \beta_{3}\right)=$ $(-4,-4)$, for a given value of the azimuthal slip-velocity parameter $\gamma_{2} \in\{0,4,8\}$. (a) The trajectories are plotted in physical, $x y z$, space together with the projection onto the $x y$ plane, constituting the boundary at $z=0$. (b) The time evolution of swimmer height $h$. (c) A plot of the associated trajectory in the phase-plane dynamical system. Note that (a) the swimmer with positive $\gamma_{2}$ rotates in a clockwise direction when viewed from above with (b) its height converging to a constant irrespective to the magnitude of $\gamma_{2}$ and (c) the time evolution of the orientation angle $\varphi$ is also independent of the rotlet dipole strength. 
opposite direction for a free-surface boundary, as discussed by $[8,9]$ for models of bacteria. Importantly, in Fig. 14, we can also observe that the rotlet dipole term just modulates the swimmer's behavior within the horizontal plane and does not change its height and orientation angle [Figs. 14(b) and 14(c)]. Hence the dynamics of the swimmer in the $x z$ plane is decoupled from the influence of the rotary dynamics.

\section{DISCUSSION AND CONCLUSION}

We have considered how an axisymmetric tangential squirmer, together with in general a rotlet dipole, behaves near a no-slip boundary and, for an asymptotically small capillary number, near a free surface, using dynamical-systems ideas to characterize the swimmer dynamics. In particular, there is a well-defined two-dimensional phase plane consisting of the height above the surface and the angle the axis of symmetry makes with the boundary, immediately demonstrating that chaotic dynamics is not possible. Nonetheless, the dynamics can be complex, as emphasized in Fig. 6, which demonstrates that a locally stable node in phase space does not necessarily entail stable global behavior due to its proximity to a saddle point.

Fortunately, for most regions of parameter space associated with boundary swimming, the dynamics is not as complicated. Boundary approach and escape for extreme angles of attack are evident from the phase-plane (e.g., Fig. 6), and the potential for extensive microswimmer boundary approach within a population of cells is consistent with the fact that, in observational studies, albumin is required to prevent human sperm sticking to microscope slides [45]. For no-slip surfaces, there is also frequently a stable fixed point, demonstrating a prediction that stable boundary swimming can occur, with a slight orientation between the swimmer and the boundary, of positive angle for a pusher and negative angle for a puller. This ability to swim stably near a surface is regularly reported and likely to feature in many observations of swimming cell behaviors, such as with sperm boundary navigation [17]. In addition, the presented results demonstrate that there is a decoupling of the dynamics perpendicular to the boundary in the presence of a rotlet dipole, so that the discussions of boundary swimming immediately apply for such swimmers, as in the context of modeling bacterial motility.

Recall that the dimensional length scale is given by $L=$ $\left(a^{2} c\right)^{1 / 3}$, where $a, c$ are the semiaxis lengths depicted in Fig. 2, with a geometric aspect ratio $A=c / a$; further note that the distance of closest approach at the fixed point in Fig. 5 is broadly the same order of magnitude across a range of parameter values. This entails that the dimensional distance of closest approach scales with $a A^{1 / 3}$ and thus we have the indication that the distance of closest approach for fixed-point boundary swimming is relatively insensitive to the geometric aspect ratio, as long as the fixed point remains stable. This is also consistent with simulations of monotrichous bacteria [23], and gives a first indication that the results observed here may be more generally representative of boundary microswimming.

It also clear in addition that fixed-point stability, as required for stable boundary swimming, is readily altered with the aspect ratio and, in particular, the beat pattern, which can be adaptively changed by a cell. Indeed the ability of biological swimmers to regulate their behavior near a no-slip surface is likely to be functionally important. One example concerns the passage of sperm through the estrous mammalian female reproductive tract, with the observation that the release of epithelial-bound sperm from tract reservoirs coincides with hyperactivation [20], which induces flagellar wave forms with lower spatial wave numbers, higher amplitudes, and asymmetric beating. In particular, it is interesting to note that hyperactivation, and its associated reduction in flagellar wave number, is predicted in modeling studies [19,25] to induce surface escape, as recapitulated here in the presented results, with the loss of fixed points to infinity on reducing the magnitude of $\beta_{3}$, representing a higher spatial wave number in the stroke. This is not the only trend in swimmer behavior that can be readily deduced by an inspection of the fixed points in Fig. 5 and is reflected in more geometrically faithful simulation studies. In particular, there is a tendency to surface approach with reduced aspect ratio [23] and the positive angle between the swimmer and the boundary for a pusher [23,25,51].

A time-reversal symmetry is readily apparent which means that pullers and pushers are dual swimmers and thus many aspects of puller dynamics can be understood from pusher dynamics. For instance, the fact pushers can switch to boundary escape behaviors with altered tangential deformations demonstrates that pullers are capable of regulating their boundary dynamics analogously. Indeed, flagellated Leishmania promastigotes are, in contrast to sperm, pulled by their flagellum, but similarly bind to and subsequently escape from midgut epithelia in their vector host, the sandfly [52]. We have demonstrated that stroke or slip velocity regulation, here at the level of the lowest modes in expansion (1), does indeed allow both pullers and pushers control over their behavior near a no-slip surfaces. Analogously, one might tentatively anticipate that ciliates and colonial algae can control their behavior near surfaces and that detailed surface patterning may alter, and yield some control over, the no-slip boundary behavior of Janus particles.

One further aspect of the time-reversal duality between pushers and pullers concerns their differences, in particular our observations of stable limit cycle dynamics. This occurs in substantial regions of parameter space, but for pullers only. Hence, by duality, pusher limit cycles are unstable for all the slip velocities we have considered. This is also consistent with the absence of limit cycles for pushers such as sperm and bacteria in observations and geometrically faithful simulations, together with the damped oscillatory decay to fixed points seen in simulations (e.g., [23,25]).

Further, note that all nondegenerate fixed points can occur in the dynamical system phase plane, so that the lowest three modes of the tangential squirmer in expansion (1) can in principle describe any phase-plane behavior aside from finetuned bifurcation dynamics. Thus the restrictions and trends we have observed concerning no-slip boundary behaviors, such as pushers do not undergo limit cycles and stably swim at positive angles relative to the boundary, may indeed be generic physically based restrictions rather than due to the necessarily limited exploration of parameter space in a predominantly simulation based study. Certainly there are no discrepancies known to the authors on comparing more geometrically faithful simulations or observational studies with the trends 
and constraints highlighted in the discussion above, which therefore can be considered as generic predictions of this modeling framework and suggest simple model squirmers can be used to explore the range of axisymmetric no-slip boundary microswimming behaviors.

We have also investigated the behavior of the axisymmetric tangential squirmer near a free surface for an asymptotically small capillary number, so that surface deformations can be neglected. In terms of scattering when stable near surface swimming does not occur, the most extensive difference was in the extent of surface residence, though a reduction in the level of scattering was also observed. These observations can be concisely understood in terms of the reduced amount of shear and thus viscous torque near the free-surface boundary compared to the no-slip scenario [53].

When attempting to consider stable near-surface swimming near a free surface with low capillary number, we in fact found that its absence is universally predicted within our modeling framework. Interestingly, the precise converse is observed for both Escherichia coli [8,41] and sperm [39]. The first of these bacterial studies also assessed interfacial properties and recorded bacterial-induced surface property changes even before trajectories were observed [41], which is consistent with substantial evidence that $E$. Coli produce surfactants [54]. Similarly, for the sperm study, sufficient surfactant was added to generate a flat drop [39] in the microscopy preparation. Thus surfactants are present, or at least most likely to be present, which has the effect of reducing surface tension, invalidating the assumption of a small capillary number regime, preventing a direct comparison of the theory presented here and observation. Nonetheless, this discrepancy does suggest that surface deformation is necessary for stable near-surface swimming as deduced, together with sufficiency though not stability, in a two-dimensional mathematical study of singularity swimmers, where the length scale of the swimmer in the 2D plane of interest is asymptotically small relative to the boundary-swimmer separation [26].

Finally, we note that our prediction of the lack of boundary accumulation near free surfaces in the absence of surfactants also represents a potential experimental test of the hydrodynamical theory studied here. Another opportunity for experimental testing would be an exploration of whether slip velocity swimmers such as Janus particles can be adjusted to undergo limit cycles in experiments. This is predicted to require a Janus particle that is a puller with a geometric polarity (e.g., $A=2$ or $A=3$ in Fig. 5) but a limited fore-aft asymmetry in the force generation to avoid a very large negative value of the force quadrupole weighting, $\beta_{3}$, which may instead induce simply stable swimming at moderate geometric polarities (e.g., $A=2, \beta_{2}>0, \beta_{3} \ll-1$ in Fig. 5). Our modeling in particular emphasizes that these tests of swimmer hydrodynamical theory would be legitimate over large regions of parameter spaces, ensuring the comparison of theory and experiment is subject to less uncertainty than quantitative tests which require detailed control over swimmer parameters.

In summary, our exploration of axisymmetric tangential squirmers as models of ciliates, colonial algae, and Janus particles has been characterized by phase-plane behaviors and simplified by a time-reversal duality. Whilst all phase-plane behaviors (except subtle bifurcations) have been observed as possible for a no-slip boundary, there are nonetheless dynamical restrictions such as pushers that do not undergo stable limit cycles. The fact all dynamical system behaviors are observed for no-slip boundaries, but these restrictions are still in place, suggests that model squirmers may be a simple framework to understand, or at least predict, generic trends and properties of axisymmetric boundary microswimming. Our observations of free-surface dynamics at low capillary number revealed that hydrodynamic changes can be understood in terms of reduced viscous torques arising on the swimmer due to the boundary, though this does not explain our predicted absence of stable near-surface swimming. This contrasts with experimental studies, though the latter are complicated by the presence of surface active substances, in turn suggesting that stable motility for finite-size swimmers near a free surface in $3 \mathrm{D}$ is necessarily contingent on surface deformation in the absence of nonhydrodynamical effects.

\section{ACKNOWLEDGMENTS}

K.I. acknowledges JSPS for support through a fellowship and a KAKENHI grant-in-aid for support. Funding support from Kyoto University's GCOE program is also acknowledged for K.I.'s visit to The Mathematical Institute, University of Oxford.
[1] C. Brennen and H. Winet, Annu. Rev. Fluid Mech. 9, 339 (1977).

[2] S. Childress, Mechanics of Swimming and Flying (Cambridge University Press, Cambridge, UK, 1981).

[3] J. S. Guasto et al., Annu. Rev. Fluid Mech. 44, 373 (2012).

[4] E. Lauga and T. Powers, Rep. Prog. Phys. 72, 096601 (2009).

[5] J. Lighthill, SIAM Rev. 18, 161 (1976).

[6] E. M. Purcell, Am. J. Phys. 1, 3 (1977).

[7] A. P. Berke, L. Turner, H. C. Berg, and E. Lauga, Phys. Rev. Lett. 101, 038102 (2008).

[8] R. DiLeonardo, D. DellArciprete, L. Angelani, and V. Iebba, Phys. Rev. Lett. 106, 038101 (2011).
[9] E. Lauga et al., Biophys. J. 90, 400 (2006).

[10] A. J. Rothschild, Nature (London) 198, 1221 (1963).

[11] H. Winet et al., J. Reprod. Fert. 70, 511 (1984).

[12] G. A. O’Toole and R. Kolter, Mol. Microbiol. 30, 295 (1998).

[13] A. B. Cunningham et al., Environ. Sci. Technol. 7, 1305 (1991).

[14] H. C. Flemming, Appl. Microbiol. Biot. 59, 629 (2002).

[15] J. Cosson et al., Cell Motil. Cytoskeleton 54, 56 (2003).

[16] D. M. Woolley, Reproduction 126, 259 (2003).

[17] P. Denissenko et al., Proc. Natl. Acad. Sci. USA 109, 8007 (2012).

[18] E. A. Gaffney et al., Annu. Rev. Fluid Mech. 43, 501 (2011).

[19] M. P. Curtis et al., J. Theor. Biol. 309, 1 (2012). 
[20] S. S. Suarez and A. A. Pacey, Hum. Reprod. Update 12, 23 (2006).

[21] D. F. Katz, J. Fluid Mech. 64, 33 (1974).

[22] M. Ramia et al., Biophys. J. 65, 755 (1993).

[23] H. Shum et al., Proc. R. Soc. A 466, 1725 (2010).

[24] L. J. Fauci and A. McDonald, Bull. Math. Biol. 57, 679 (1995).

[25] D. J. Smith et al., J. Fluid Mech. 621, 289 (2009).

[26] D. Crowdy et al., J. Fluid Mech. 681, 24 (2011).

[27] Y. Or et al., SIAM J. Appl. Dyn. Syst. 10, 1013 (2011).

[28] R. Zargar, A. Najafi, and M. F. Miri, Phys. Rev. E 80, 026308 (2009).

[29] S. E. Spagnolie and L. Lauga, J. Fluid Mech. 700, 105 (2012).

[30] I. Llopis and I. Pagonabarraga, J. Non-Newtonian Fluid Mech. 165, 946 (2010).

[31] J. R. Blake, J. Fluid Mech. 46, 199 (1971).

[32] M. J. Lighthill, Commun. Pure Appl. Math. 5, 109 (1952).

[33] M. Kreutz et al., J. Eukaryot. Microbiol. 59, 548 (2012).

[34] W. F. Paxton et al., Chem. Eur. J. 11, 6462 (2005).

[35] V. Magar and T. J. Pedley, J. Fluid Mech. 539, 93 (2005).

[36] S. Michelin and E. Lauga, Phys. Fluids 22, 111901 (2010).

[37] T. Ishikawa et al., J. Fluid Mech. 568, 119 (2006).

[38] T. Goto et al., J. Biomech. Sci. Eng. 5, 329 (2010).
[39] S. Boryshpolets et al., Theriogenology 79, 81 (2013).

[40] N. Ricci et al., Linmol Oceangr. 36, 1178 (1991).

[41] L. Lemelle et al., J. Bacteriol. 192, 6307 (2010).

[42] S. H. Lee et al., J. Fluid Mech. 93, 705 (1979).

[43] C. Pozrikidis, A Practical Guide to Boundary Element Methods with the Software Library BEMLIB (CRC Press, Boca Raton, 2002).

[44] A. A. Evans et al., Phys. Fluids 23, 111702 (2011).

[45] D. J. Smith and J. R. Blake, Math. Sci. 20, 74 (2009).

[46] C. Pozrikidis, Boundary Integral and Singularity Methods for Linearized Viscous Flow (Cambridge University Press, Cambridge, UK, 1992).

[47] J. R. Blake, Proc. Cambr. Philos. Soc. 70, 303 (1971).

[48] J. D. Klein, J. Colloid Interface Sci. 261, 379 (2003).

[49] J. P. Armitage and R. M. Macnab, J. Bacteriol. 169, 514 (1987).

[50] K. A. Morehouse et al., J. Bacteriol. 187, 1695 (2005).

[51] D. Giacche, T. Ishikawa, and T. Yamaguchi, Phys. Rev. E 82, 056309 (2010).

[52] P. A. Bates, Curr. Opin. Microbiol. 11, 340 (2008).

[53] J. Ferracci et al., PLoS ONE 8, e75238 (2013).

[54] R. Zhang et al., Proc. Natl. Acad. Sci. USA 107, 288 (2010). 